\title{
Ripple formation on a particle bed sheared by a viscous liquid. Part 1 . Steady flow
}

\author{
By F. CHARR U ${ }^{1}$ AND E. J. HINCH ${ }^{2}$ \\ ${ }^{1}$ IMFT, Allée C. Soula, 31400 Toulouse, France \\ ${ }^{2}$ Department of Applied Mathematics and Theoretical Physics, University of Cambridge, \\ Wilberforce Road, Cambridge CB3 0WA, UK
}

(Received 15 December 2004 and in revised form 19 July 2005)

A new approach to studying ripple formation in steady flow is proposed, based on a model for the erosion and deposition of the particles. Higher shear stresses at the crests lead to a loss of particles from there, which can suppress the instability induced by fluid inertia. This model accounts for recent observations that ripples do not grow when the fluid viscosity is increased, a behaviour not present in the classical approach based on an algebraic dependence of the particle flux on the bottom shear stress.

\section{Introduction}

Sand ripples under water can be created by steady flows, as in rivers and channels, as well as by oscillating flows, such as those induced by surface waves in shallow water. The currently accepted mechanism for ripple formation is purely hydrodynamic, and is the same in both cases: it is based on an instability of the flat sand bed due to fluid inertia. In the fluid above a small-amplitude deformation of the bed, inertia is responsible for a phase lag between the bottom shear stress and the wavy bed. The out-of-phase component of shear stress slowly drags the particles from the troughs up to the crests, hence the instability. The phase lag of the shear stress over a wavy boundary was first calculated by Benjamin (1959) for the case of a uniform shear flow, and its crucial role in sand ripple formation first discussed by Kennedy (1963). However, the potential flow theory used by Kennedy was unable to determine the phase lag, which was a free parameter. The hydrodynamic instability mechanism, which is described in more detail in the following section, holds for any steady flow, either turbulent (Richard 1980; Sumer \& Bakioglu 1984; Colombini 2004) or viscous (Charru \& Mouilleron-Arnould 2002). All wavenumbers are unstable, but the stabilizing effect of gravity dominates for high wavenumbers, hence the occurrence of a long-wave instability with a cut-off wavenumber and a most amplified wavenumber which can be compared to observations.

However up to now, no experiment has clearly proved that the above instability mechanism is the dominant one, for several reasons (Raudkivi 1997). Very few growth-rate measurements are available (Betat, Frette \& Rehberg 1999), and the observed ripple lengths display large scatter (Yalin 1985), which can be partly attributed to ripple coalescence which occurs at the early stages of the growth. Moreover, several experiments indicate that wavelengths mainly depend on the particle diameter, with only slight dependence on the fluid flow (Coleman \& Eling 2000), whereas stability theories predict that the wavelength should scale on a viscous length (Sumer \& Bakioglu 1984; Charru \& Mouilleron-Arnould 2002). From the 


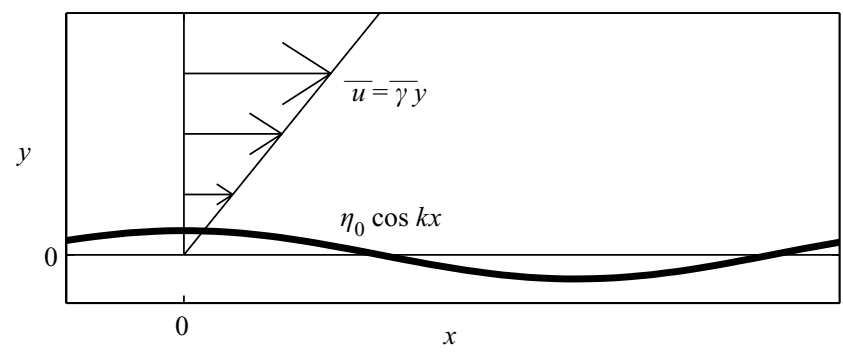

FIGURE 1. Sketch of the fluid layer over a sinusoidal bed, and the base flow.

modelling point of view, the main reason for the failure of theoretical predictions may be that, apart from the turbulence problem, the dynamics of the particle is poorly accounted for through an algebraic law relating the particle flux to the bottom shear stress. Moreover, the modelling of the stabilizing effect of gravity involves a numerical coefficient which is not well-known, and to which the most-amplified wavenumbers is very sensitive (Richard 1980; Colombini 2004). In addition to the above points, recent experiments in a Couette-flow device have shown that when fluid viscosity is increased, ripples disappear for steady flow (Mouilleron 2002). This observation cannot be understood within the explanation sketched in the previous paragraph.

The aim of this paper is to propose a new approach to bed stability based on a model for the erosion and deposition of particles rolling or sliding over the fixed bed, a situation usually referred to as 'bedload transport'. This model brings out a stabilizing effect which is absent from the previous models, and which accounts for the above observation that increasing the fluid viscosity suppresses the ripples. This study is divided in two parts: the present first part is devoted to steady flow, and the following second part to oscillating flow (Charru \& Hinch 2006). This part is organized as follows. The viscous fluid flow over a sinusoidal bed is given in $\S 2$. The erosion-deposition model is discussed in $\S 3$. Section 4 is devoted to the calculation of the density of moving particles and the growth rate for long waves. A summary and discussion is given in $\S 5$.

\section{Fluid flow}

We consider a fluid layer with density $\rho$, viscosity $\mu$ and thickness $h$, lying between an erodible bed and a moving upper wall (figure 1). The erodible bed is assumed to be perturbed sinusoidally as $\eta=\eta_{0} \cos k x$, with wavenumber $k$ and amplitude $\eta_{0}$. Following previous studies and in agreement with observations, we assume that the time scale of the fluid flow is much shorter than the time scale of the bed evolution. Thus the fluid flow can be calculated as if the wavy bottom were fixed, by considering the flow as the superposition of a base flow $\bar{u}$ over a flat bed, and a disturbance $(u, v)$ induced by the wavy bottom.

The base flow is given by $\bar{u}=\bar{\gamma} y$ with shear rate $\bar{\gamma}=U_{w} / h$, where $U_{w}$ is the upper wall velocity. For small bed slope $\left(k \eta_{0} \ll 1\right)$, the disturbance flow can be calculated from the linearized momentum equations and boundary conditions. This problem has been solved by Charru \& Hinch (2000), and so we only sketch its solution. The problem involves three length scales: the inverse wavenumber $k^{-1}$, the thickness of 

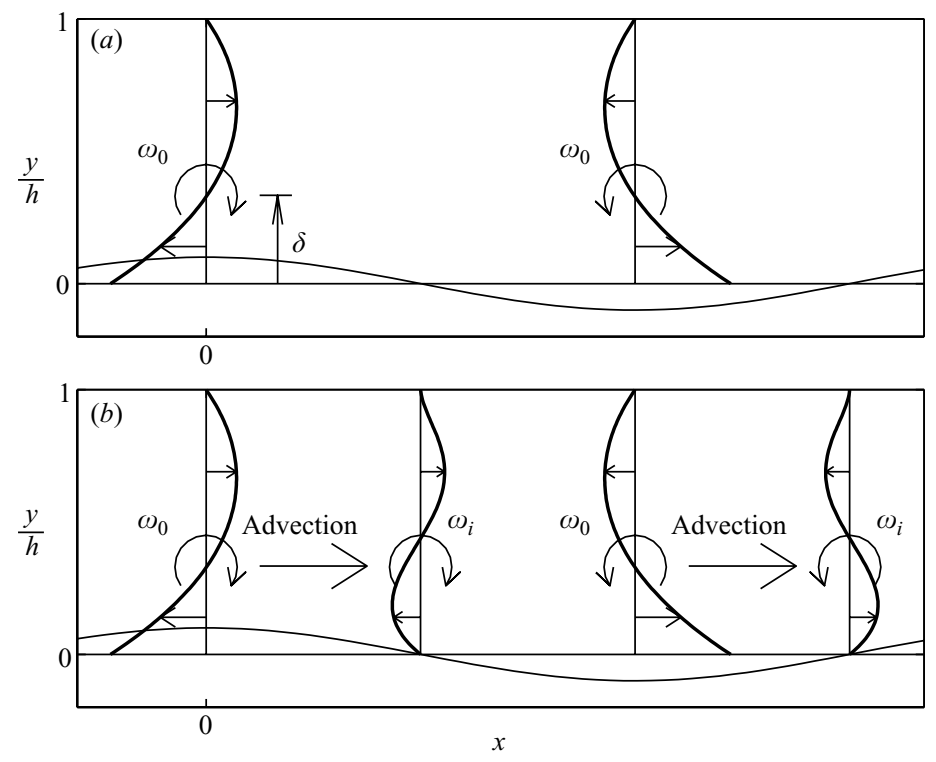

FiguRE 2. (a) Typical velocity disturbance $u_{0}$ in phase with the wavy bottom, and corresponding vorticity disturbances $\omega_{0}$ and penetration height $\delta$. $(b)$ Same as $(a)$ but for the inertially induced out-of-phase components $u_{i}$ (magnified by a factor 100) and $\omega_{i}$.

the fluid layer $h$, and the viscous length $l_{v}$ defined as

$$
l_{v}=\left(\frac{v}{k \bar{\gamma}}\right)^{1 / 3} \text {. }
$$

First we consider Stokes flow over a wavy bottom. The disturbance flow has to ensure the no-slip condition on the perturbed boundary, at which the base flow $\bar{u}=\bar{\gamma} \eta$ is non-zero. Thus, negative (resp. positive) longitudinal velocities $u$ are created near the crests (resp. the troughs), and these diffuse upwards as shown in figure 2(a). The corresponding vorticity disturbances $\omega_{0}$ diffuse over a penetration height $\delta$, which scales as the smallest of the three length scales

$$
\delta \sim \min \left(k^{-1}, h, l_{v}\right)
$$

In the case of Stokes flow, the bottom shear stress disturbance is in phase with the bottom: it is maximum at the top of the crests, and minimum at the bottom of the troughs.

For small fluid inertia, advection by the base flow of the vorticity disturbance $\omega_{0}$ creates an out-of-phase vorticity component $\omega_{i}$, with an associated velocity disturbance $u_{i}$ as shown in figure $2(b)$. The no-slip condition forces the velocity $u_{i}$ to decrease to zero near the bottom, where the sign of $\omega_{i}$ reverses. Thus an outof-phase bottom shear stress component is created, which shifts the maximum of the shear stress slightly upstream of the crests.

The bottom shear stress is the sum of the base flow component $\mu \bar{\gamma}$ and the disturbance $\mu \gamma$, where the shear-rate disturbance can be written as

$$
\gamma=\epsilon_{0} \bar{\gamma}\left(\cos k x-\epsilon_{i} \sin k x\right) \text {. }
$$

In this equation, the $\epsilon_{0}$-term corresponds to the dominant Stokes correction due to the bottom waviness, and the $\epsilon_{i}$-term corresponds to inertia effects due to advection. The 


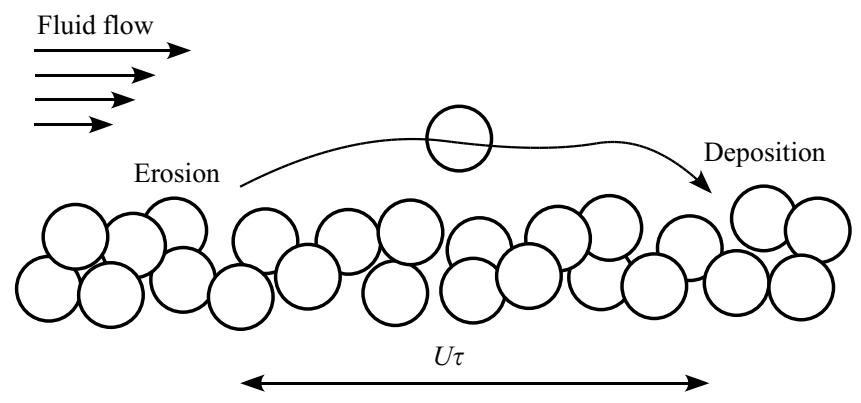

FIGURE 3. Sketch of the erosion and deposition phenomena, and length $U \tau$ of typical saltation flights.

parameters $\epsilon_{0}$ and $\epsilon_{i}$ in general involve the Airy functions Ai and Bi and integrals of these functions. However these parameters always remain close to simple expressions corresponding to the three following asymptotic regimes:

(i) shallow viscous regime $\left(h \ll k^{-1}\right.$ and $h \ll l_{v}$, i.e. $\left.\delta \sim h\right)$

$$
\epsilon_{0}=\frac{4 \eta_{0}}{h}, \quad \epsilon_{i}=\frac{k h}{120} \frac{\rho U_{w} h}{\mu} ;
$$

(ii) deep viscous regime $\left(k^{-1} \ll l_{v}\right.$ and $k^{-1} \ll h$, i.e. $\left.\delta \sim k^{-1}\right)$

$$
\epsilon_{0}=2 k \eta_{0}, \quad \epsilon_{i}=\frac{\rho \gamma}{4 k^{2} \mu}
$$

(iii) boundary layer regime $\left(l_{v} \ll k^{-1}\right.$ and $l_{v} \ll h$, i.e. $\left.\delta \sim l_{v}\right)$

$$
\epsilon_{0}=\frac{1.06 \sqrt{3}}{2} k \eta_{0}\left(\frac{\rho \gamma}{k^{2} \mu}\right)^{1 / 3}, \quad \epsilon_{i}=\frac{1}{\sqrt{3}} .
$$

Finally, all that is needed for the following long-wave analysis of the particle motion is the bottom shear rate (2.3) along with the long-wave parameters (2.4).

\section{An erosion-deposition model}

Now consider a bed of particles, of diameter $d$ and density $\rho_{p}$, sheared by a fluid flow (figure 3). The condition for a particle at the flat bed surface to be set into motion is that the hydrodynamic force acting on it, of order $\mu \bar{\gamma} d^{2}$, exceeds a fraction of the apparent weight of the particle, of order $\left(\rho_{p}-\rho\right) g d^{3}$, i.e. that the dimensionless bottom shear stress,

$$
\bar{\theta}=\frac{\mu \bar{\gamma}}{\left(\rho_{p}-\rho\right) g d},
$$

often referred to as the Shields number (Raudkivi 1998), exceeds a threshold value $\theta_{t 0}$. For fluid flow over a sinusoidal bottom, the local dimensionless bottom shear stress can be defined from the local shear rate $(\bar{\gamma}+\gamma)$, where $\gamma$ is given by (2.3). Normalizing with the threshold Shields number $\theta_{t 0}$ for future convenience, we set

$$
\Theta=\bar{\Theta}+\epsilon_{0}\left(\Theta^{(0)}+\epsilon_{i} \Theta^{(1)}\right),
$$


where $\bar{\Theta}$ is the normalized Shields number for the flat bed, and $\Theta^{(0)}$ and $\Theta^{(1)}$ are the wavy-bed Stokes and inertial corrections, defined as

$$
\bar{\Theta}=\frac{\bar{\theta}}{\theta_{t 0}}, \quad \Theta^{(0)}=\bar{\Theta} \cos k x, \quad \Theta^{(1)}=-\bar{\Theta} \sin k x .
$$

We now turn to the erosion-deposition model. Let $\dot{n}_{e}$ and $\dot{n}_{d}$ be the number of particles eroded and deposited, respectively, per unit time and unit bed area, and let $q=n U$ be the particle flux, where $n$ the number of moving particles per unit bed area and $U$ is the mean particle velocity. Then the number density of moving particles obeys the following conservation equation:

$$
\frac{\partial n}{\partial t}=-\dot{n_{d}}+\dot{n_{e}}-\frac{\partial q}{\partial x} .
$$

The deposition rate $\dot{n_{d}}$ can be modelled from the observation that moving particles experience saltation flights with typical flight time $\tau$ (van Rijn 1984; Charru, Mouilleron-Arnould \& Eiff 2004). This flight time is assumed here to be proportional to the settling time $d / V_{S}$, where $V_{S}$ is the Stokes settling velocity of a single particle,

$$
\tau=\frac{d}{c_{d} V_{S}} \quad \text { with } \quad V_{S}=\frac{\left(\rho_{p}-\rho\right) g d^{2}}{18 \mu},
$$

where $c_{d}$ is a coefficient. During one flight time $\tau, n$ particles are expected to stop per unit area, so that the deposition rate can be expressed as

$$
i_{d}=\frac{n}{\tau} .
$$

The erosion of a particle depends on the hydrodynamic force acting on it, i.e. on the bottom shear rate. At leading order, the erosion rate can be expected to be proportional to the excess shear rate $\left(\bar{\gamma}+\gamma-\gamma_{t}\right)$, where $\gamma_{t}$ is the threshold shear rate. This erosion rate is likely to depend weakly on the number of moving particles, but this dependence is neglected here. Introducing the saltation time $\tau$, the erosion rate can be written as

$$
\dot{n}_{e}=\frac{c_{e}}{\tau d^{2}}(\Theta-1),
$$

where $c_{e}$ is a coefficient.

Finally, the mean particle velocity $U$, which is involved in the particle flux $q=n U$, is assumed to be proportional to the fluid velocity $(\bar{\gamma}+\gamma) d$ at the distance $d$ above the bed surface. Thus, $U$ can be written as

$$
U=\frac{d}{\tau} c_{u} \Theta
$$

where $c_{u}$ is a coefficient.

Choosing the particle diameter $d$ and the jump time $\tau$ as the unit length and time, respectively, we introduce the dimensionless space, time and wavenumber as

$$
X=\frac{x}{d}, \quad T=\frac{t}{\tau}, \quad K=k d,
$$

and the dimensionless density $N$ of moving particles and particle flux $Q$ as

$$
N=n d^{2}, \quad Q=q \tau d=c_{u} \Theta N .
$$


Then the erosion-deposition equation (3.4) becomes

$$
\frac{\partial N}{\partial T}=-N+c_{e}(\Theta-1)-\frac{\partial Q}{\partial X}, \quad \text { with } \quad Q=c_{u} \Theta N .
$$

The above model was inspired by experiments reported in Charru et al. (2004), which, from measurements of particle fluxes and saltation flights, allowed the determination of all coefficients. For silicon oil with viscosity $\mu=20$ Pas and acrylic beads with diameter $0.58 \mathrm{~mm}$, the settling time was $d / V_{S}=0.27 \mathrm{~s}$ and the coefficients were found to be

$$
\theta_{t 0}=0.12, \quad c_{e}=0.055, \quad c_{d}=0.067, \quad c_{u}=3.3 .
$$

\section{Ripple formation}

\subsection{Density of mobile particles}

In this section, the erosion-deposition equation (3.11), together with equations (3.2)(3.3) for $\Theta$, is solved for the particle density $N$, using a long-wave expansion $(K \ll 1)$ of the density $N$ and the flux $Q$,

$$
\begin{aligned}
& N=\bar{N}+\epsilon_{0}\left(N^{(0)}+K N^{(1)}+K^{2} N^{(2)}+\cdots\right), \\
& Q=\bar{Q}+\epsilon_{0}\left(Q^{(0)}+K Q^{(1)}+K^{2} Q^{(2)}+\cdots\right) .
\end{aligned}
$$

Since both parameters $\epsilon_{i}$ and $K$ involve the wavenumber $k$, we assume that they are of the same order of magnitude, so that

$$
\frac{\epsilon_{i}}{K}=\frac{\operatorname{Re}}{120} \frac{h}{d}=O(1)
$$

Moreover, since the time-derivative in (3.11) only gives rise to exponentially decreasing terms with time scale $\tau$, we ignore transients and focus on the asymptotic steady solution.

For the flat bed $\left(\epsilon_{0}=0\right)$, the particle density corresponds to an equilibrium between erosion and deposition, leading to a linear dependence of $\bar{N}$ on $\bar{\Theta}$, and a quadratic dependence for $\bar{Q}$ :

$$
\bar{N}=c_{e}(\bar{\Theta}-1), \quad \bar{Q}=c_{e} c_{u} \bar{\Theta}(\bar{\Theta}-1) .
$$

For the wavy bed, the shear stress is higher on the crests, where erosion is therefore higher. Then the local erosion-deposition equilibrium implies higher particle density, hence the $O\left(\epsilon_{0} K^{0}\right)$ correction

$$
N^{(0)}=c_{e} \bar{\Theta} \cos K X .
$$

The particle flux is also higher on the crests because the shear stress and the particle density are higher there, hence the correction $Q^{(0)}=c_{u}\left(\bar{N} \Theta^{(0)}+N^{(0)} \bar{\Theta}\right)=c_{e} c_{u} \bar{\Theta}(2 \bar{\Theta}-1)$.

For the investigation of higher-order effects, we first ignore fluid inertia $\left(\epsilon_{i}=0\right)$. At $O\left(\epsilon_{0} K^{1}\right)$, the divergence of the disturbance flux $Q^{(0)}$ induces an accumulation of particles on the lee side of the ripples, with density

$$
N^{(1)}=c_{e} c_{u} \bar{\Theta}(2 \bar{\Theta}-1) \sin K X .
$$

Since there is no further erosion at this order for $\epsilon_{i}=0$, these particles settle, as illustrated in figure 4(a), inducing a drift of the wavy interface with positive velocity, without growth or decay. The particle flux associated with the transport of $N^{(1)}$ 
(a)

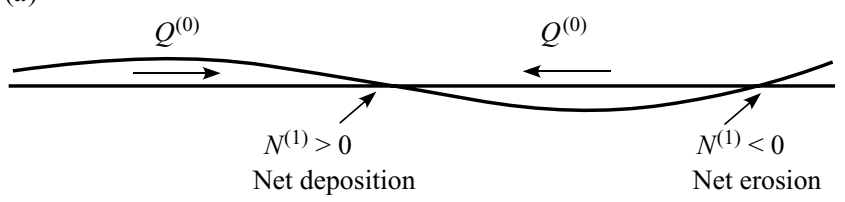

(b)

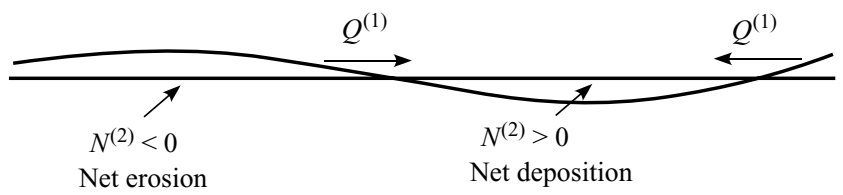

FiguRE 4. Sketch of the particle flux and particle density disturbances for zero fluid inertia $\left(\epsilon_{i}=0\right)$. (a) Leading-order disturbance $Q^{(0)}$, whose divergence lead to net deposition $N^{(1)}$ and wave drift. $(b)$ Higher-order correction $Q^{(1)}$ induced by $N^{(1)}$, whose divergence lead to net deposition $N^{(2)}$ and wave decay.

by the flow is $Q^{(1)}=c_{u} N^{(1)} \bar{\Theta}$. At $O\left(\epsilon_{0} K^{2}\right)$, the divergence of this flux induces an accumulation of moving particles in ripple troughs, with density

$$
N^{(2)}=-c_{e} c_{u}^{2} \bar{\Theta}^{2}(2 \bar{\Theta}-1) \cos K X .
$$

Since there is no erosion as at the previous order, these particles settle in the troughs, as illustrated in figure $4(b)$. Thus, when fluid inertia is ignored, the disturbance decays and the flat bed is stable. This result is quite different from that predicted by previous stability analyses, which, considering that the particle flux simply adjusts to the local shear rate through a Bagnold-type law $Q=Q(\Theta)$, predict neutral disturbances if the bottom shear stress is in phase with the wavy bed. In contrast our new model of a layer of mobile particles develops phase shifts from the rates of deposition and erosion, so that the higher shear rates at the crests can lead to a loss of particles from the crest.

For small but non-zero inertia $\left(\epsilon_{i} \neq 0\right)$, an out-of-phase shear-stress component arises, as discussed in $\S 2$, pointing towards the crests. This shear stress, of order $\epsilon_{0} \epsilon_{i} \sim \epsilon_{0} K$, contributes to erosion, and the local erosion-deposition equilibrium brings an inertial contribution to the correction $N^{(1)}$ calculated above, equation (4.6), given by

$$
N_{i}^{(1)}=-\frac{\operatorname{Re}}{120} \frac{h}{d} c_{e} \bar{\Theta} \sin K X .
$$

This correction does not contribute to the wave velocity, because there is no net deposition with the local balance of erosion with deposition. The particle flux associated with it is given by $Q_{i}^{(1)}=c_{u}\left(N_{i}^{(1)} \bar{\Theta}+\left(\epsilon_{i} / K\right) \bar{N} \Theta^{(1)}\right)$. At $O\left(\epsilon_{0} K^{2}\right)$, the divergence of this flux induces an accumulation of particles on ripple crests, with density

$$
N_{i}^{(2)}=\frac{\operatorname{Re}}{120} \frac{h}{d} c_{e} c_{u} \bar{\Theta}(2 \bar{\Theta}-1) \cos K X .
$$

These particles correspond to a net deposition, so that the effect of fluid inertia is to increase the amplitude of the bed disturbance. 
Now combining the crest-erosion term $N^{(2)}$ and the crest-deposition term $N_{i}^{(2)}$, it appears that if fluid inertia is small $\left(N_{i}^{(2)}+N^{(2)}<0\right.$ on crests), crest-erosion dominates and the bed disturbance decays, whereas if fluid inertia is strong enough, the inverse is true and the bed disturbance grows. Both $N_{i}^{(2)}$ and $N^{(2)}$ involve the shear rate, in the Reynolds number $R e$ and the Shields number $\bar{\Theta}$, but the marginal stability condition does not depend on it. This condition corresponds rather to a critical value of the ratio of $R e$ and $\bar{\Theta}$, which is related to the Galileo number (a Reynolds number based on the sedimentation velocity rather than the externally imposed shear)

$$
G a=\left(\frac{d}{h}\right)^{2} \frac{R e}{\theta_{t 0} \bar{\Theta}}, \quad \text { where } G a=\frac{\rho_{p}-\rho}{\rho} \frac{\rho^{2} g d^{3}}{\mu^{2}} .
$$

Marginal stability then corresponds to a critical value $G a_{c}$ of the Galileo number, given by

$$
G a_{c}=\frac{120 c_{u}}{\theta_{t} 0}\left(\frac{d}{h}\right)^{3} .
$$

Thus, for $G a>G a_{c}$ (typically large particle diameter or small viscosity), the destabilizing effect of fluid inertia overcomes the stabilizing effect of crests erosion, so that the flat bed is unstable (as long as the restoring effect of gravity, to be considered in the next section, is ignored). For $G a<G a_{c}$, the flat bed is stable for any bed shear stress.

\subsection{Effect of gravity}

On a wavy bed, the component of gravity parallel to the bed pulls the particles towards troughs with a force $\left(\rho_{p}-\rho\right) g \partial_{x} \eta$ per unit volume. This force has two effects: it modifies the threshold Shields number $\theta_{t 0}$ for the flat bed, and it modifies the velocity of the mobile particles. The modified threshold can be taken as (Soulsby \& Whitehouse 1997)

$$
\theta_{t}=\theta_{t 0}\left(1+\frac{\partial_{x} \eta}{\tan \chi}\right)
$$

where $\chi$ is the static angle of friction of the particles (angle of repose), and is about $32^{\circ}$ (Bagnold 1973). This modifies the erosion term $c_{e}(\Theta-1)$ in (3.11) to

$$
c_{e}\left(\Theta-1+\epsilon_{0} \frac{k h}{4 \tan \chi} \sin K X\right) .
$$

The above threshold correction induces at $O\left(\epsilon_{0} K^{1}\right)$ a correction to the particle density,

$$
N_{g}^{(1)}=c_{e} \frac{h}{4 d \tan \chi} \sin K X,
$$

whose transport by the base flow gives the first contribution of gravity to $Q^{(1)}$.

The second contribution of gravity to $Q^{(1)}$ arises from the particle velocity due to the component of gravity parallel to the bed. This velocity is expected to be proportional to the Stokes falling velocity $-V_{S} \partial_{x} \eta$, so that the particle velocity (3.8) has to be modified to

$$
U=\frac{d}{\tau} c_{u} \Theta+U_{g}, \quad \text { with } \quad U_{g}=\frac{d}{\tau} c_{g} \epsilon_{0} k h \sin K X,
$$

where $c_{g}$ is a coefficient. The transport of $\bar{N}$ with the falling velocity $U_{g}$ brings the second contribution of gravity. At $O\left(\epsilon_{0} K^{2}\right)$, the divergence of the gravity flux gives 
the following correction to the particle density:

$$
N_{g}^{(2)}=-c_{e} \frac{h}{d}\left(\frac{c_{u}}{4 \tan \chi} \Theta+c_{g}(\Theta-1)\right) \cos K X .
$$

The first term on the right-hand side arises from the threshold correction (4.12), and the second from the velocity correction (4.15). They have the same order of magnitude and correspond to particle deposition in troughs, so that gravity is stabilizing, as expected.

\subsection{Wave velocity and growth rate}

The wave velocity and growth rate of the bed disturbance are related to the net deposition rate $\dot{n_{d}}-\dot{n}_{e}$, which from the above results is

$$
\tau d^{2}\left(\dot{n}_{d}-\dot{n}_{e}\right)=\epsilon_{0}\left(-\partial_{X} Q^{(0)}+k d N_{g}^{(1)}\right)+\epsilon_{0}(k d)^{2}\left(N^{(2)}+N_{i}^{(2)}+N_{g}^{(2)}\right) .
$$

The first two terms on the right-hand side will contribute to the propagation velocity, whereas the other three will give the growth rate. Multiplying this net deposition rate by the particle volume and dividing by the volume fraction $\phi$ of the particles in the bed, which is assumed to be uniform, we obtain the time-derivative of the bed surface,

$$
\frac{\partial \eta}{\partial t}=\frac{\pi d^{3}}{6 \phi}\left(\dot{n}_{d}-\dot{n}_{e}\right)
$$

The wave velocity $c$ and growth rate $\sigma$ can be obtained from the above equation with $\eta=\eta_{0} \mathrm{e}^{\sigma t} \cos k(x-c t)$. The wave velocity is

$$
c=\frac{d}{\tau} \frac{2 \pi}{3 \phi}\left(c_{e} c_{u} \frac{d}{h} \bar{\Theta}(2 \bar{\Theta}-1)+\frac{c_{e}}{4 \tan \chi}\right) .
$$

The first term arises from the divergence of $Q^{(0)}$, and the second, from $N_{g}^{(1)}$. Both terms are positive so that the bed disturbance propagates downstream.

The growth rate is

$$
\sigma=\frac{1}{\tau} \frac{2 \pi}{3 \phi} c_{e} c_{u}(k d)^{2}\left(\left(\frac{G a \theta_{t 0}}{120} \frac{h^{2}}{d^{2}}-c_{u} \frac{d}{h}\right) \bar{\Theta}^{2}(2 \bar{\Theta}-1)-\frac{\bar{\Theta}}{4 \tan \chi}-c_{g}(\bar{\Theta}-1)\right) .
$$

The first term on the right-hand side is the only destabilizing term, arising from fluid inertia; the second one corresponds to the stabilizing effect of crest erosion, and the last two correspond to the stabilizing effect of gravity. All these terms scale as the square of the wavenumber, so that all wavenumbers are either stable or unstable. If the Galileo number, $G a$, is lower than the critical one, $G a_{c}$, defined by (4.11), fluid inertia cannot overcome crest erosion, and the flat bed is stable whatever the Shields number. In the opposite case, $G a>G a_{c}$, fluid inertia dominates crest erosion, and there exists a critical Shields number $\theta_{c}$ beyond which the bed is unstable. If the stabilizing effect of gravity is weak compared to the net destabilizing effect of fluid inertia and crest erosion, then $\theta_{c}=\theta_{t 0}$ and the bed is unstable as soon as particle move; if gravity is strong enough, the critical Shields number $\theta_{c}$ is higher than $\theta_{t}$, and there exists a Shields-number range, $\theta_{t 0}<\bar{\theta}<\theta_{c}$, in which the flat bed is stable.

\section{Summary and discussion}

The present study discusses ripple formation on a bed sheared by a steady viscous flow, using a more accurate description of the particle motion than previous ones. As described in the introduction section, the usual explanation of ripple formation reduces the particle dynamics to an algebraic law for the particle flux as a function 
of the bottom shear stress. Then, the phase lag of the bottom shear stress induced by fluid inertia implies instability; and zero phase lag implies zero growth rate (as long as the gravity component parallel to the wavy bed is ignored). Here, the particle motion was derived from a conservation equation for the density of mobile particles. This equation involves in particular an erosion-rate term and a deposition-rate term, which have been modelled simply using two time scales: a settling time for the deposition rate, and the inverse shear rate for the erosion rate. This model was first introduced in Charru et al. (2004) from experiments in a Couette-flow device, and its reliability has been discussed there in connection with previous numerical and experimental results.

The main result obtained from the erosion-deposition model is the existence of a stabilizing term which does not depend on fluid inertia. This term arises from the fact that on the crests, a higher shear stress implies more mobile particles, which are transported by the flow and then settle in troughs. This phenomenon, although easily understandable physically, is not taken into account in previous models, even in the viscous resuspension model by Charru \& Mouilleron-Arnould (2002). The net result of the competition between the destabilizing inertia and the stabilizing crest erosion depends on the Galileo number. For small Galileo number (typically small particle diameter or large viscosity), crest erosion dominates and the bed is stable, whatever the Shields number. For large Galileo number, fluid inertia dominates and the bed is unstable beyond a critical Shields number $\theta_{c}$. If the net stabilizing effect is weak, the critical Shields number is equal to the threshold $\theta_{t 0}$; otherwise there exists a Shields-number range for which the flat bed is stable.

The existence of a critical Galileo number can explain why Mouilleron (2002) observed that ripples disappear when the fluid viscosity is increased: these experiments in an annular Couette device showed that ripples grow for viscosity $\mu$ lower than $19 \times 10^{-3} \mathrm{Pas}$, corresponding to $G a>2.2$, whereas for viscosity $\mu$ greater that $26 \times 10^{-3} \mathrm{~Pa}$ s, corresponding to $G a<1.1$, the bed remained flat for several days in the range of Shields-number explored $(\theta<1)$. For the ratio of the depth of fluid to the diameter of the grains used in Mouilleron's experiments, $h / d=18.4$, the critical Galileo number predicted by (4.11) is 0.53 , which is lower than the observed one by a factor about two. The difference may be accounted for by the stabilizing gravity effect which could not be evaluated accurately, since it involves the coefficient $c_{g}$, as yet unknown. Thus, it can be said that the present theory accounts for the observation of the stabilisation of the bed when the fluid viscosity is increased, and predicts at least the right order of magnitude of the critical Galileo number. The existence of a critical Galileo number may also explain why Leighton \& Acrivos (1986) do not report instabilities in their Couette resuspension-flow experiments: their Galileo number was in the range 0.001-0.054, whereas the critical Galileo number (4.11) was of the order of unity.

Finally, the present long-wave analysis cannot predict any wavenumber selection since the predicted growth rate (4.20) scales as the square of the wavenumber. However, this analysis is no longer valid for wavelengths shorter than the fluid depth. While a detailed analysis for arbitrary values of the wavenumber must await a future study, we can easily see how the expression for the growth rate (4.20) will change in the limit of viscous short waves, $k h \gg 1$, by using equation (2.5) for $\epsilon_{0}$ and $\epsilon_{1}$. The destabilizing term in (4.20) proportional to $G a$ will be divided by $(k h)^{2}$, the next term for the stabilizing effect of crest erosion will be multiplied by $k h$, and the final two terms for the stabilizing effect of gravity will remain unchanged. These changes make short waves stable. Hence the maximum growth rate is expected to occur when $k h=O(1)$. 


\section{REFERENCES}

Bagnold, R. A. 1973 The nature of saltation and of "bed-load" transport in water. Proc. R. Soc. Lond. A 332, 473-504.

Betat, A., Frette, V. \& Rehberg, I. 1999 Sand ripples induced by water shear flow in an annular channel. Phys. Rev. Lett. 83, 88-91.

Benjamin, T. B. 1959 Shearing flow over a wavy boundary. J. Fluid Mech. 6, 161-205.

Charru, F. \& Hinch, E. J. 2000 'Phase diagram' of interfacial instabilities in a two-layer Couette flow and mechanism for the long-wave instability. J. Fluid Mech. 414, 195-223.

Charru, F. \& Hinch, E. J. 2006 Ripple formation on a particle bed sheared by a viscous liquid. Part 2. Oscillating flow. J. Fluid Mech. 550, 123-137.

Charru, F. \& Mouilleron-Arnould, H. 2002 Instability of a bed of particles sheared by a viscous flow. J. Fluid Mech. 452, 303-323.

Charru, F., Mouilleron-Arnould, H. \& Eiff, O. 2004 Erosion and deposition of particles on a bed sheared by a viscous flow. J. Fluid Mech. 519, 55-80.

Coleman, S. E. \& Eling, B. 2000 Sand wavelets in laminar open-channel flows. J. Hydraul. Res. 38, 331-338.

Colombini, M. 2004 Revisiting the linear theory of sand dune formation. J. Fluid Mech. 502, 1-16.

Kennedy, J. F. 1963 The mechanics of dunes and antidunes in erodible-bed channels. J. Fluid Mech 16, 521-544.

Leighton, D. \& Acrivos, A. 1986 Viscous resuspension. Chem. Engng Sci. 41, 1377-1384.

Mouilleron, H. 2002 Instabilités d'un milieu granulaire cisaillé par un fluide. Thèse de Doctorat, Université Paul Sabatier, Toulouse, France.

Raudkivi, A. J. 1997 Ripples on stream bed. J. Hydraul. Engng 123, 58-64.

RAUdKIVI, A. J. 1998 Loose Boundary Hydraulics. A. A. Balkema, Rotterdam.

Richards, K. J. 1980 The formation of ripples and dunes on an erodible bed. J. Fluid Mech. 99, 597-618.

VAN RIJN, L. C. 1984 Sediment transport, part I: bed load transport. J. Hydraul. Engng 110, $1431-1456$.

Soulsby, R. L. \& Whitehouse, R. J. S. 1997 Threshold of sediment motion in coastal environments. In Proc. Australasian Coastal Engng and Ports Conf., Christchurch, pp. 149-154.

Sumer, B. M. \& BaKioglu, M. 1984 On the formation of ripples on an erodible bed. J. Fluid Mech. 144, 177-190.

YALIN, M. S. 1985 On the determination of ripple geometry. J. Hydraul. Engng 111, 1148-1155. 